\title{
Rol de los dispositivos para entrenar caninos empleados para la seguridad y defensa nacional: una revisión sistemática de la literatura de los últimos veinte años
}

\section{Role of canine training devices used for homeland security and defense: \\ a systematic review of the literature over the past twenty years \\ Papel dos dispositivos de treino canino utilizados para a segurança e defesa nacional: uma revisão sistemática da literatura ao longo dos últimos vinte anos}

Fecha de recepción: 2020/I2/0I | Fecha de evaluación: 2021/08/23 | Fecha de aprobación: 2021/09/03

Jorge Ulises Rojas-Guevara

I*M.V. Especialista en Seguridad Pública, Especialista en Servicio de Policía, Ms.C., Ph.D. Teniente Coronel (r) de la Policía Nacional de Colombia Director del Research Center Working Dogs $®$ ORCID 0000-0003-4925-5365. jorge.rojask9team@gmail.com

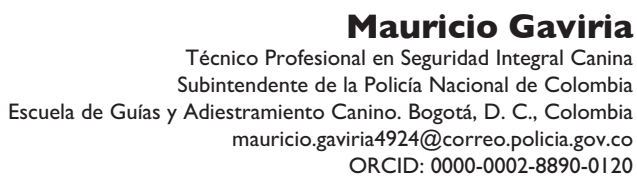

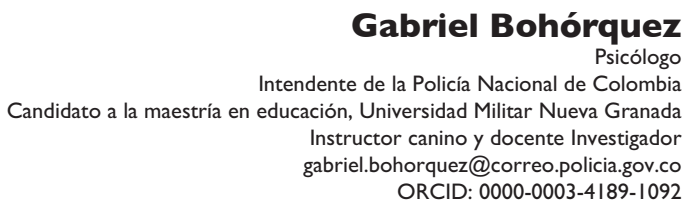

David Orlando Gutiérrez Guauta

Técnico Profesional en Seguridad Integral Canina Intendente de la Policía Nacional de Colombia Escuela de Guías y Adiestramiento Canino david.gutierrez7357@correo.policia.gov.co Código ORCID: 0000-000I-9438-3740

Para citar este artículo / To reference this article / Para citar este artigo: Rojas-Guevara, J. U., Bohórquez, G., Gaviria, M., \& Gutiérrez, D. (2022). Rol de los dispositivos para entrenar caninos empleados para la seguridad y defensa nacional: una revisión sistemática de la literatura de los últimos veinte años. Revista Criminalidad, 64(I), I55- I75. https://doi.org/I0.4774I// 7943 I 08.337

\section{Resumen}

El entrenamiento de los caninos de trabajo emplea diversos mecanismos de condicionamiento, los cuales permiten un rendimiento posterior superior, contrarrestando el sistema de drogas ilícitas, las organizaciones criminales, los grupos armados organizados (GAO) y residuales (GAOr), garantizando la seguridad y convivencia ciudadana en Colombia. Por lo anterior, se propone un enfoque cualitativo empleando una revisión sistemática de la literatura, con el objetivo de analizar el rol de la tecnología y aparatos para adiestrar caninos detectores, entre los años 2000 y 2020 dentro de las bases de datos Scopus, Elsevier y Scielo. Como resultados, se observa un aumento en la producción de artículos entre los años 2000 y 2019 (pasando de seis artículos a 86 , respectivamente). Además, dentro de las herramientas empleadas en los estudios se encuentran las cajas; clickers; collares electrónicos y carruseles, los cuales discriminan el olor, utilizando sistemas de refuerzo, con diferencias dependiendo del tipo de estudio, el número de animales y el objetivo de entrenamiento. Como conclusión, es necesario desarrollar prototipos adecuados según las necesidades de entrenamiento en cada contexto, continuando con estudios que integren efectivamente los estímulos y los sistemas de recompensa para impactar los resultados en el rendimiento del perro de trabajo.

\section{Palabras clave}

Seguridad ciudadana, tecnología (fuente: Tesauro Criminológico - Instituto de Investigación Interregional de Crimen y Justicia de las Naciones Unidas - UNICRI). Rendimiento canino, refuerzo positivo (fuente: autores). 


\section{Abstract}

The training of working canines employs various conditioning mechanisms, allowing for superior subsequent performance, counteracting the illicit drug system, criminal organisations, organised armed groups (GAO) and residual armed groups (GAOr), guaranteeing the safety and security and civic coexistence in Colombia. Therefore, a qualitative approach is proposed by means of a systematic review of the literature between the years 2000 and 2020 within the databases of Scopus, Elsevier and Scielo, with the objective of analysing the role of technology and devices to train detecting canines. As a corollary, there is evidence of an increase in the production of articles from the years 2000 to 2019 (from 6 articles to 86). Additionally, among the tools used in the studies, there are boxes; clickers; electronic collars and carousels, which discriminate odor, using reinforcement systems, with differences depending on the type of study, the number of animals and the training objective. As a conclusion, it is necessary to develop suitable prototypes according to the training needs in each context, continuing with studies that effectively integrate stimuli and the reinforcement systems to impact the results in the performance of the working dog.

\section{Keywords}

Civic security, technology (source: Criminological Thesaurus - United Nations Interregional Crime and Justice Research Institute - UNICRI). Canine performance, positive reinforcement (source: authors).

\section{Resumo}

A formação de caninos de trabalho emprega vários mecanismos de condicionamento, que permitem um desempenho posterior superior, contrariando o sistema de drogas ilícitas, organizações criminosas, grupos armados organizados (GAO) e grupos armados residuais (GAOr), garantindo a segurança dos cidadãos e a coexistência na Colômbia. Por conseguinte, é proposta uma abordagem qualitativa, utilizando uma revisão sistemática da literatura, com o objetivo de analisar o papel da tecnologia e dos dispositivos de treino de cães detectores, entre 2000 e 2020 nas seguintes bases de dados: Scopus, Elsevier e Scielo. Os resultados mostram um aumento na produção de artigos entre 2000 e 2020 (de 6 artigos para 86, respectivamente). Além disso, entre as ferramentas utilizadas nos estudos, existem caixas, clickers, coleiras eletrônicas e carrosséis, que discriminam o cheiro, utilizando sistemas de reforço, com diferenças em função do tipo de estudo, do número de animais e do objetivo de formação. Em conclusão, é necessário desenvolver protótipos adequados de acordo com as necessidades de treino em cada contexto, continuando com estudos que integrem eficazmente estímulos e sistemas de recompensa para impactar os resultados no desempenho do cão de trabalho.

\section{Palavras-chave}

Segurança dos cidadãos, tecnologia (fonte: Thesaurus Criminológico - Instituto Inter-regional de Pesquisa em Crime e Justiça das Nações Unidas - UNICRI). Desempenho canino, reforço positivo (fonte: autores).

\section{Introducción}

La seguridad y defensa nacional comprende un conjunto de acciones coordinadas y ejecutadas por distintos órganos del Estado para garantizar la estabilidad de un país, incluidos en el marco de convenios de cooperación nacional e internacional; por ende, dentro de estas actividades se contemplan aquellas relacionadas con la lucha contra el sistema de drogas ilícitas, la neutralización de amenazas que pongan en riesgo la vida de los ciudadanos y el resguardo del orden. Por lo anterior, el rol que cumplen los organismos de seguridad del Estado es fundamental para mantener la convivencia y la paz. Así mismo, el papel que cumple la Policía Nacional es fundamental para mantener la seguridad y convivencia ciudadana, dentro de los cuales encontramos los caninos detectores de sustancias, siendo necesario que discriminen el olor (Prada-Tiedemann et al., 2019a), además de garantizar los criterios de selección, entrenamiento, certificación y rendimiento (PradaTiedemann et al., 2019b).

En Colombia, el conflicto armado vivido durante los últimos sesenta años ha dejado huellas profundas en la sociedad, que han obligado a las autoridades a desarrollar estrategias avanzadas de inteligencia, con el fin de combatir consecuencias como el narcotráfico y 
el uso de explosivos como medios para desestabilizar la paz y el orden. Al revisar las cifras más relevantes sobre la cantidad de hectáreas cultivadas de coca en el país, así como las estrategias para combatirlas como la Erradicación Manual Forzosa (EMF) y la Aspersión Aérea (AA) (Sarmiento et al., 2020), se encuentra que hubo un incremento del $140 \%$ comparativamente entre el año 2019 respecto a $201 \mathrm{I}$; mientras tanto, la EMF se ha implementado en proporción a la cantidad de hectáreas cultivadas, pasando de $35.20 \mathrm{I}$ en $20 \mathrm{II}$ a 94.605 en 2019 , lo cual representa un $93 \%$ de crecimiento; por el contrario, la AA dejó de realizarse a partir del año 2016 según las fuentes oficiales.

Por otra parte, las estadísticas del Observatorio de Drogas de Colombia (ODC) indican que la cocaína y la marihuana son dos de las más importantes sustancias psicotrópicas incautadas en el territorio nacional. Con respecto a la primera, se observa que en el año 2017 fueron decomisadas cerca de 324 toneladas; en años posteriores esta cifra disminuyó un $58 \%$, ubicándose en 137 toneladas en el año 2019. Igualmente, las labores de detección de marihuana lograron quitar de circulación unas 306 toneladas en promedio durante el periodo de los años $201 \mathrm{I}$ a 2019 (ODC, 2019). Por consiguiente, el aporte que generan los caninos en la lucha contra el narcotráfico (Villarreal et al., 2018), la detección de restos humanos (Rojas-Guevara et al., 2020b), el tráfico de especies en peligro de extinción y otros delitos asociados (Rojas-Guevara et al., 2020a) es crucial, por ello, el entrenamiento mediante dispositivos de apoyo es clave para lograr mejores rendimientos y posiblemente disminuir las cifras delictivas e impactar las organizaciones criminales y los grupos armados organizados (GAO).

\section{Marco teórico}

En los delitos relacionados con el narcotráfico se encuentran problemas sociales como el homicidio y la desaparición (Córdoba-Parra et al., 2020) casos en los cuales los perros de trabajo desempeñan un rol fundamental para la búsqueda y recuperación de elementos materiales probatorios y evidencia física en el lugar de los hechos (Deruyter et al., 2020; Raymer et al., 2020), además de los caninos detectores de residuos biológicos (Cáceres \& Rojas-Guevara, 2020). En tal sentido, las cifras de la Secretaría Distrital de Seguridad, Convivencia y Justicia (SDSCJ) en la ciudad de Bogotá señalan que existe un aumento del 19,5\% en el año 2020 con respecto al 2019 en homicidios (SDSC], 2020). A su vez, delitos como el tráfico de especies de fauna y flora silvestre, son uno de los más importantes en el territorio nacional, dado que las cifras parciales para 2020 reflejan que existe un total de 3.083 incautaciones, donde el $76 \%$ corresponde a reptiles, $15 \%$ aves y $9 \%$ mamíferos, ante esta realidad el uso de perros para detectar la comercialización ilegal de animales suele ser útil y efectiva (SDSC), 2020).

La Policía Nacional de Colombia (PNC), como parte integral del cuerpo armado de naturaleza civil, garante de la seguridad y convivencia, cuenta con diferentes unidades especializadas que le permiten combatir problemas sociales como el tráfico de drogas y el uso de explosivos con fines terroristas, apoyándose para ello en el entrenamiento de caninos de razas de trabajo específicas; así, conforma binomios (guíaperro) cuyo objeto es detectar de manera eficiente y efectiva las potenciales amenazas a la convivencia y seguridad ciudadana. Como parte integral de la PNC, la Escuela de Guías y Adiestramiento Canino (ESGAC) ha sido la unidad responsable de entrenar binomios en distintas tareas que incluyen el rastro de personas, detección de narcóticos, explosivos, papel moneda, fauna silvestre (Sánchez et al., 20I4), búsqueda y rescate de personas, ubicación de restos humanos, cuidados terapéuticos e hidrocarburos (PNC, 2015; Ochoa et al., 2020).

Con el fin de seguir aportando a este propósito desde la institución policial, se ha venido desarrollando avances para mejorar los dispositivos que se emplean en el proceso de entrenamiento de los caninos, contribuyendo a la identificación y detección de sustancias o materiales controlados por la ley (drogas, explosivos, bebidas alcohólicas y productos de tabaco y cigarrillos), discriminación de olores humanos (Rojas-Guevara, et al., 2020b) y detección de especies de fauna silvestre (Rojas-Guevara et al., 2020a). Estos esfuerzos por desplegar mejoras en los dispositivos han sido motivados por las falencias encontradas en los actuales instrumentos utilizados, entre estos las cajas para entrenamiento, clickers, collares y similares, puesto que no logran integrar de manera efectiva elementos clave para desarrollar el comportamiento esperado en el perro, según las funciones para las cuales está destinado.

Por otra parte, es fundamental documentar la manera en que se pueden integrar los sistemas de refuerzo para el aprendizaje de los caninos, puesto que ante la amplia gama de dispositivos de entrenamiento que existen, es importante articularlos entre sí, considerando, además, sus niveles de complejidad, contexto de uso y propósito, con el fin de orientar a los manejadores sobre cómo mejorar los procesos 
de entrenamiento. Al no contar con los recursos formativos adecuados para lograr una discriminación y detección efectiva en los caninos, se estaría afectando los resultados y el rendimiento contra el narcotráfico, la identificación de explosivos, las labores de búsqueda y rescate, entre otras actividades clave para el resguardo de la convivencia y seguridad ciudadana.

Con base en este contexto, el objetivo del estudio es analizar el rol de los dispositivos para el entrenamiento de caninos empleados en actividades de seguridad y defensa nacional, con el fin de poder comparar sus ventajas y desventajas para luego sugerir, con base en los hallazgos, las prestaciones y características idóneas que debería tener un dispositivo en el contexto de la detección y discriminación de olores asociados con el entrenamiento. Adicionalmente, a fines del presente artículo se considera conceptualmente un dispositivo como toda aquella herramienta, sea tecnológica o no, que apoye el proceso de entrenamiento para la discriminación o detección de olores provenientes de sustancias nocivas (drogas), explosivos, labores de búsqueda y rescate. Entre estas, las más comunes son: (a) cajas; (b) clickers; (c) collares electrónicos y (d) carruseles.

\section{Método}

El objeto de estudio se aborda desde un enfoque cualitativo, el cual es definido por Creswell y Poth (2016), como el conjunto de técnicas y herramientas que se emplean para comprender la realidad en un contexto natural no determinista, es decir, busca analizar los fenómenos con base en un proceso reflexivo propio del paradigma interpretativo (Goldkuhl, 20I2). Por lo tanto, los hallazgos no pretenden explicar el rol de los dispositivos en entrenamiento de caninos empleados en actividades de seguridad y defensa nacional desde una perspectiva estadística o cuantitativa, sino analizar estos dispositivos en función de lo publicado en la literatura.

Para lograr este propósito se emplea la revisión sistemática de la literatura (SLR, por sus siglas en inglés) tomando como referencia el proceso recomendado por algunos autores relevantes en este ámbito. Si bien se ha empleado la SLR en diferentes campos de estudio como la medicina (Higgins et al., 2019), la ingeniería (Kitchenham et al., 2009), así como las ciencias sociales y ambientales (Mengist et al., 2020), las aplicaciones y utilidad de este tipo de método proporcionan al investigador una perspectiva amplia sobre la temática de interés, dado que permite recopilar sistemáticamente el conocimiento de numerosos artículos científicos que se publican periódicamente.

Como justificación del método, Rodríguez, Zafra y Ortega (2015) han señalado la utilidad de las SLR para exponer los avances en la literatura, sobre todo para instituciones como la Policía Nacional de Colombia (PNC), la cual viene realizando esfuerzos significativos para potenciar el conocimiento en el ámbito de la seguridad y defensa, la investigación, la innovación y el aprovechamiento de las tecnologías de la información y comunicación (TIC) en pro de mejorar la gestión de la entidad. Por ello, se transforman en una forma de generar nuevas ideas por medio del contraste de los hallazgos de diferentes autores, sirviendo de base para realizar estados del arte en campos específicos del saber. De acuerdo con lo anterior, Beltrán (2005) manifiesta que la SRL puede asumirse como un estudio observacional y retrospectivo que integra fuentes de información secundaria para combinarlos y producir conocimiento útil asociado a una misma pregunta de investigación; por lo tanto, argumenta que ante la cantidad de documentos que se generan constantemente, el desarrollo SLR en temas específicos ofrece a la comunidad científica la oportunidad de mantenerse actualizado en relación con los nuevos avances de un campo de estudio sintetizando aquellos más relevantes.

Con el fin de dar una visión general del proceso, se toman como referencia los lineamientos sugeridos por Snyder (2019), quien define cuatro fases principales en la SLR, a saber: (a) diseño: en esta etapa se analiza la contribución científica que aportará la $S L R$ y la necesidad de realizarla. Además, se identifica la audiencia potencial, el propósito general y las preguntas de investigación que orientarán el estudio. Por otra parte, se establece el método más apropiado según los objetivos planteados; (b) ejecución: se define un plan de búsqueda para seleccionar los artículos, así como los criterios de inclusión y exclusión que se emplearán para la identificación de las publicaciones, lo que a su vez se convertirá en un mecanismo para garantizar la calidad del proceso de investigación; (c) análisis: en esta fase se determina el impacto de las publicaciones a través del contenido del resumen, palabras clave y demás elementos de interés para el investigador; adicionalmente, se define la información específica que se necesitará para responder las preguntas del estudio; y (d) redacción: una vez compilada, organizada y seleccionada toda la información pertinente se inicia el proceso reflexivo para comunicar los resultados los cuales deben ser comunicados claramente al lector. 


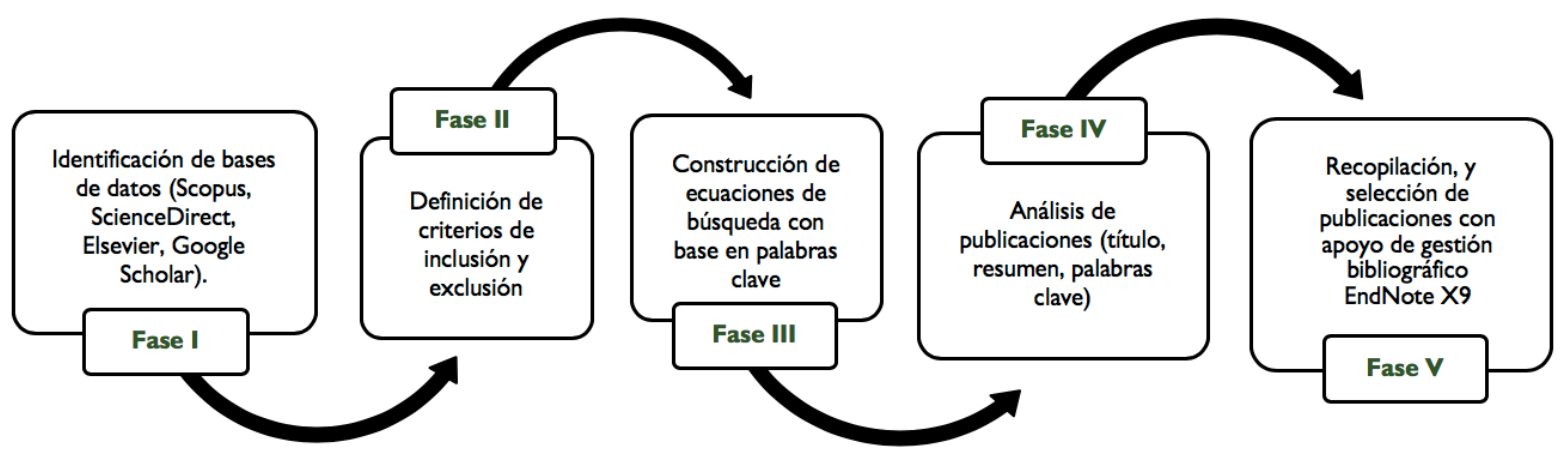

Figura I

Procedimiento empleado en la SLR

Fuente: Autores, 2021.

Tabla I

Resultados de la ecuación de búsqueda en bases de datos

\begin{tabular}{|c|c|c|c|}
\hline Bases de datos & Ecuación & Resultado & Selección \\
\hline Scopus, Elsevier, Science Direct & TITLE-ABS-KEY (training AND & 758 & 65 \\
\hline
\end{tabular}

En cuanto al procedimiento secuencial para la realización de la SLR, se inició identificando las bases de datos potenciales donde se pudiesen encontrar los artículos de interés, así como la pertinencia de utilizar el motor de búsqueda Scholar Google como apoyo. El criterio para seleccionar las bases de datos señaladas en la figura I se basó en el impacto y la relevancia que tienen en el ámbito científico, dado que muchos son los autores y revistas que publican en este tipo de recursos. Asimismo se emplearon los siguientes aspectos como criterios de inclusión: artículos relacionados con las palabras clave training, method, dog, detection y device publicados en los últimos veinte años tanto en idioma inglés como español; por el contrario, se excluyeron: (a) artículos sobre temáticas asociadas con el comportamiento canino, capacidad de discriminación y condición física de perros de trabajo con el fin de reducir el espectro de búsqueda; (b) se descartaron capítulos de libros, artículos de conferencias y literatura gris. Por su parte, para la búsqueda en inglés se consideraron los términos training, method, dog, detection y device, utilizando operadores boleanos AND/OR/AND NOT, que facilitaron la identificación de artículos de interés.

\section{Resultados}

A continuación se muestran los resultados de la primera búsqueda en las diferentes bases de datos mencionadas con las ecuaciones y los resultados preliminares que arrojaron (tabla I).

En función de los resultados de búsqueda en Scopus, se encontró que los autores con mayor impacto en el campo de métodos de entrenamiento canino son Miklósi $(h$-index $=57)$, Mills $(h$-index $=31$ ), Bennett ( $h$-index $=27$ ) y Bentosela $(h$-index $=16$ ); además, se clasifican como los investigadores con mayor producción científica en este eje temático específico $(n>18)$. Así mismo, la figura 2 muestra el promedio anual de documentos publicados por autor según los datos reportados por Scopus.

Se advierte en la figura 3 un notorio interés de la comunidad científica en los temas asociados con el entrenamiento canino, puesto que se observa un crecimiento sostenido en los últimos veinte años; en términos cuantitativos, la cifra de las publicaciones en el año 2020 es siete veces superiores a lo que se difundía en el año 2000.

Realizar una revisión sistemática de la literatura consolida y condensa la pesquisa relevante dispersa, convirtiéndola en el preámbulo ideal hacia un tema novedoso como los dispositivos utilizados para entrenar caninos detectores en la seguridad y defensa nacional. Además, se encuentra que a pesar de que existen artículos que generan nuevo conocimiento en torno a dicha temática, es un inicio que posiblemente impulsará la dinámica para entrenar caninos detectores bajo nuevos estándares de trabajo empírico, en las especialidades del servicio policial. Así mismo, este 
trabajo permite a investigadores, académicos y estudiantes de varias disciplinas conocer los últimos estudios asociados, los cuales se analizarán según dos categorías: (I) sistemas de refuerzo y dispositivos para entrenamiento canino y (2) patentes asociadas.

\section{Discusión}

\section{Sistemas de refuerzo y dispositivos para entrenamiento canino}

Se considera que un sistema de refuerzo se usa para lograr comportamientos apropiados del animal, ante la respuesta del material objeto por detectar, contribuyendo además a mantener esta conducta en el largo plazo para que no se extinga (Bentosela et al., 2008), por tal motivo, estos han sido analizados empíricamente en la literatura desde hace décadas.
Estudios como los de Mcintire y Collen (1967) hallaron que el rendimiento de los perros bajo programas de entrenamiento disciplinado estuvo sujeto a los tipos de refuerzo empleados. En primer lugar, se encontró que el elogio verbal por sí solo no fue suficiente para mantener una respuesta constante en el animal; por otra parte, la inclusión de caricias restableció el rendimiento, y se concluye que la estimulación táctil es un refuerzo primario.

Profundizando en esta temática, Mclntire (1968) halló que en el entrenamiento de perros tanto comerciales como militares se emplean los refuerzos positivos como caricias, comida, juguetes, entre otros, o negativos, implicando procedimientos disciplinarios previos que permiten restringir la conducta del animal durante el adiestramiento; así como los estímulos que señalan el momento en el que se aplican las consecuencias. En tal sentido, los refuerzos sociales aversivos y positivos se consideran

Figura 2

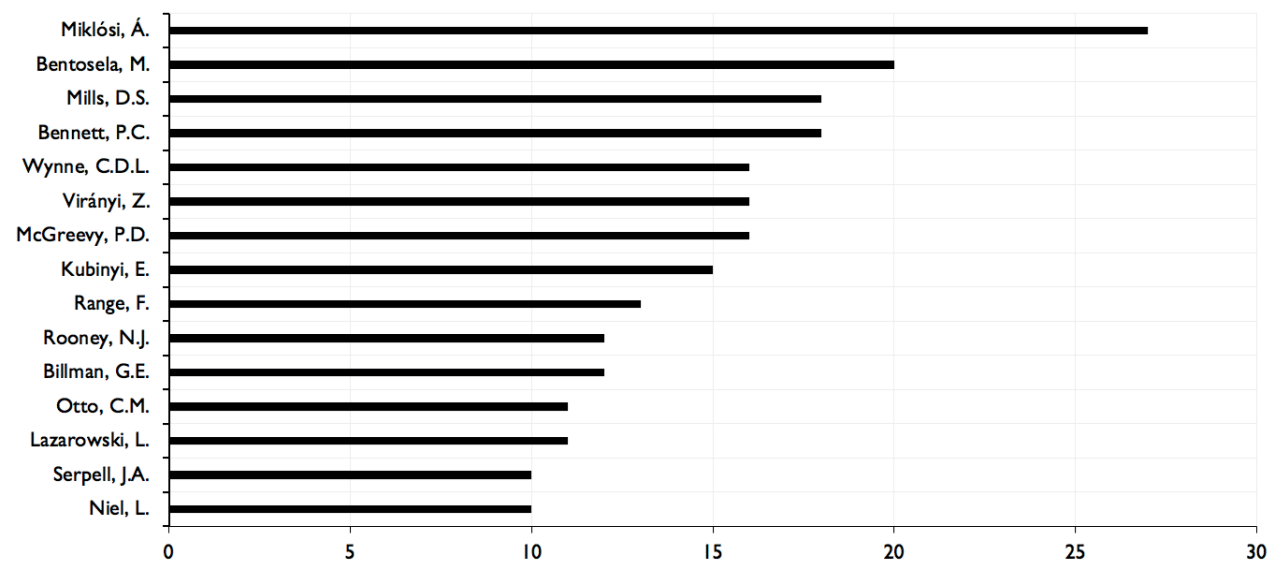

Documentos publicados por autor Scopus

Fuente: Scopus, 2020.

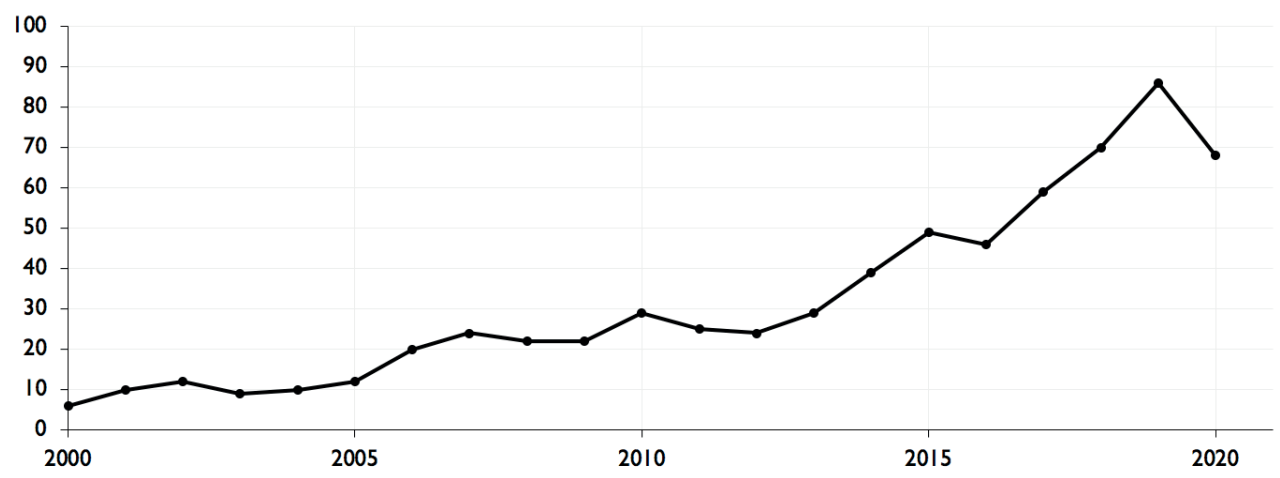

Figura 3

Evolución del número de publicaciones asociadas con entrenamiento canino

Fuente: Scopus, 2020. 
más adecuados para caninos en entornos familiares, mientras que en otros contextos se pueden utilizar los verbales como reforzadores secundarios asociados a una estimulación aversiva. Posteriormente, Fonberg y Kostarczyk (1980) manifestaron que el refuerzo social pertenece al sistema de recompensa general, incluyendo el sonoro, el táctil y el alimenticio. Así mismo, sugieren que aspectos fisiológicos como el funcionamiento de las amígdalas y el hipotálamo en el animal son aspectos clave para otorgar valor a las recompensas y se deben considerar al momento del entrenamiento, dado que un daño en estos sistemas podría provocar alteraciones no deseadas en el comportamiento del perro.

En esta misma línea de análisis, Browne et al. (20I I) estudiaron la comunicación entre perros y humanos, demostrando que estos animales son sensibles a las señales que se les dan, entre estas la señalización, miradas y vocalizaciones; en función de ello, sugieren que resulta viable emplear este tipo de mecanismos para entrenarlos. Sin embargo, los mismos autores encontraron una variación significativa entre los tiempos de refuerzo y la latencia del entrenador para entregar las instancias de recompensa, sean estas primarias o secundarias, concluyendo que los retrasos en el refuerzo pueden generar retroalimentación incorrecta en el perro, dado que estos son altamente receptivos a las señas del humano.

En años siguientes, evidencia reportada por Browne et al. (20l3) indica que el retraso del refuerzo tiene un impacto en el aprendizaje de algunos caninos al realizar nuevas tareas. No obstante, las características de la formación y la entrega oportuna del refuerzo parecen no ser consistentes en todos los perros, por lo tanto, concluyen que el momento preciso para dar el refuerzo es una variable que condiciona el éxito del entrenamiento; en tal sentido, en la interacción entre el animal, el entrenador y el dispositivo es importante considerar estos aspectos con el fin de incrementar el éxito del procedimiento. Desde esta perspectiva, se considera que el refuerzo es una variable crítica que influye considerablemente en el comportamiento del perro, por ello el dispositivo que se utilice para el entrenamiento debe tomar en cuenta la relevancia de este componente.

Por otra parte, en el contexto de los sistemas de refuerzo, es relevante comprender los tipos de condicionamiento empleados en el entrenamiento canino: operante y clásico; con respecto al primero, es un aprendizaje que se genera de acuerdo con las consecuencias del comportamiento, es decir, con este método el sujeto conoce los efectos de sus acciones y actúa en respuesta a ello (Blackman, 2017). El estudio de la conducta bajo esta perspectiva fue impulsado por Skinner (197I), quien experimentó con animales para observar las relaciones entre el comportamiento y los estímulos del ambiente, y encontró que era posible explicar dichas conductas y hacer predicciones sensatas sobre los cambios observados.

El dispositivo de experimentación empleado para analizar este fenómeno, conocido como la caja de Skinner, consistía en un espacio cerrado donde se colocaba el animal, en este caso ratas, con unas palancas que al activarlas emitían una señal visual (luz) o auditiva (sonido), otorgando una recompensa alimenticia, por lo tanto, los animales lograban una asociación entre sus acciones y el premio; posteriormente se modificó el procedimiento con el propósito de que los animales revirtieran el aprendizaje, haciendo que la activación de las palancas no proporcionara el alimento, observando que las ratas volvieran al estado inicial (Skinner, 197I). La figura 4 muestra un ejemplo de esta estructura experimental.

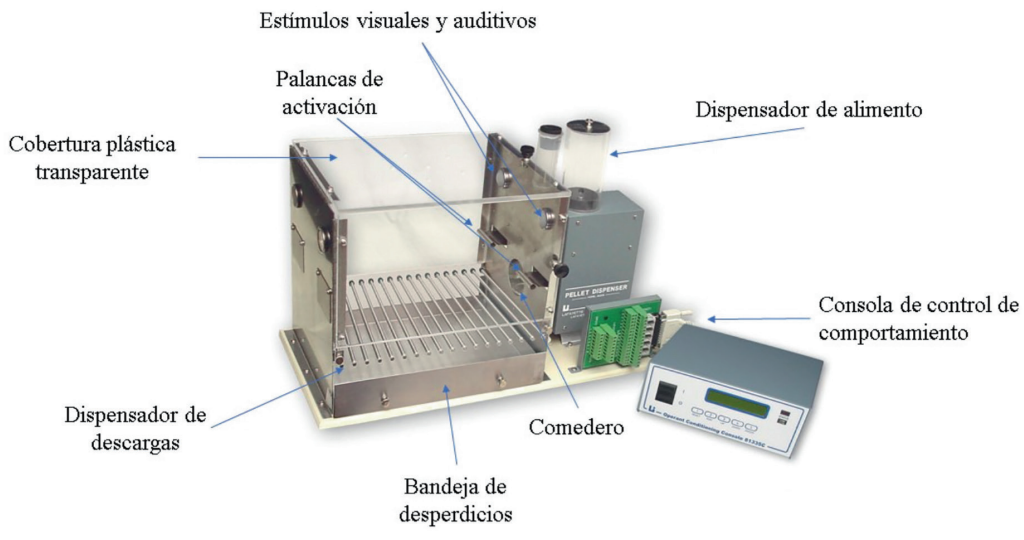

Figura 4

Caja de condicionamiento operante marca Lafayette (USA) modelo $84025 * \mathrm{C}$ 
Tabla 2

Categorización de sistemas de refuerzo

\begin{tabular}{|c|c|c|c|}
\hline $\begin{array}{l}\text { Categoría } \\
\text { de refuerzo }\end{array}$ & Definición & Método de entrenamiento & $(\%) *$ \\
\hline Positivo & $\begin{array}{l}\text { El comportamiento del animal aumenta } \\
\text { luego de la exposición a un estímulo } \\
\text { subjetivamente agradable. }\end{array}$ & $\begin{array}{c}\text { Elogios verbales } \\
\text { Recompensa alimenticia } \\
\text { Acariciar } \\
\text { Jugar } \\
\text { clicker (refuerzo positivo condicionado) }\end{array}$ & $\begin{array}{l}96 \\
84 \\
83 \\
68 \\
16\end{array}$ \\
\hline Negativo & $\begin{array}{l}\text { El comportamiento aumenta luego de la } \\
\text { exposición a un estímulo probablemente } \\
\text { repugnante para el animal. }\end{array}$ & $\begin{array}{l}\text { Retirar la atención } \\
\text { Retener recompensas de comida } \\
\text { Restricción física (ej., mantener posición) }\end{array}$ & $\begin{array}{l}45 \\
10 \\
12\end{array}$ \\
\hline Castigo positivo & $\begin{array}{l}\text { El comportamiento en este caso se } \\
\text { reduce posterior a la presentación de un } \\
\text { estímulo potencialmente repugnante. }\end{array}$ & $\begin{array}{c}\text { Reprensión verbal } \\
\text { Corrección física (sacudir o bofetear) } \\
\text { Sonido no verbal como distractor }\end{array}$ & $\begin{array}{c}64 \\
37 \\
5\end{array}$ \\
\hline
\end{tabular}

(*) Porcentaje de respondientes que utilizan este tipo de métodos.

Fuente: Blackwell et al. (2008).

Seguidamente, en el condicionamiento clásico propuesto por Pavlov (1926) en los experimentos realizados con perros, el organismo logra asociar los nuevos estímulos con respuestas biológicas, por ejemplo, salivación, sudoración, temor; por lo tanto, no se produce aprendizaje, sino que surge un comportamiento preestablecido en función de la señal que se está observando. La teoría se fundamenta en cuatro elementos: (a) estímulo incondicionado (EI), que es aquel que produce una respuesta natural sin haber sido aprendida; (b) respuesta incondicionada (RI), que constituye la reacción ante el El; (c) estímulo condicionado (EC), que corresponde al elemento neutro que provoca una respuesta que se asocia a otro estímulo; y (d) respuesta condicionada (RC), que es el comportamiento aprendido frente al estímulo.

De acuerdo con el experimento de Pavlov (1926), se utiliza la carne para que el perro salive, creando una RI, por otra parte, al introducir un elemento neutro como un sonido sin asociarlo, no hará que se produzca cambio alguno, sin embargo, al emparejar el sonido con la carne, este se convertirá en un estímulo condicionado lo que provocará en el animal una RC que será la salivación. Esta explicación resulta relevante para tratar de comprender el entrenamiento canino más allá del dispositivo que se esté empleando y el sistema de refuerzo escogido por el ser humano.

Según lo manifestado por Gabrielsen (2017) y según los postulados de Skinner (197I) y Pavlov (1926), estos han sido ampliamente empleados en los procesos de entrenamiento canino desde sus inicios hasta la actualidad; además, realiza un señalamiento relevante sobre la utilización del dispositivo conocido como clicker argumentando que cuando este se aplica no resulta claro determinar quién es el entrenador o aprendiz, el sujeto y el objeto, dicho de otro modo, el que controla y el controlado. El motivo de esta reflexión radica en que la elección del método de entrenamiento puede desempeñar un rol crítico en el éxito de la relación de dualidad perro-humano.

Estudios realizados por Blackwell et al. (2008) revelaron que los métodos de entrenamiento aplicados tienen una asociación significativa con las conductas potencialmente indeseables en los perros, informando a través de pruebas estadísticas no paramétricas Kruskal-Wallis $(X 2=12.865, d f=$ $3, p<0.01$ ). Así mismo, señalan que en todos los casos analizados el refuerzo positivo se asoció con puntuaciones medias bajas en la escala de conductas violentas en el animal, como la búsqueda de atención $(0,33)$, miedo o evitación $(0,18)$ y agresión $(0,10)$. Seguidamente se presenta, en la tabla 2 , la categorización de los sistemas de refuerzo empleados por los entrenadores, para luego comprender su relación con los comportamientos del canino.

En definitiva, Blackwell et al. (2008) determinaron que existe una relación entre comportamientos indeseables y el método de entrenamiento, siendo el refuerzo positivo o aquellos que no utilizan el castigo los que menos ocurrencia de este tipo de conductas provocan. En concordancia con lo anterior, Hiby et al. (2004) también encontraron que los sistemas de refuerzo positivo suelen tener una mayor efectividad para evitar efectos adversos en el comportamiento e 
incrementar su capacidad para aprender. Al respecto, los métodos de entrenamiento que encontraron en los estudios se distribuían en tres categorías: (a) basados en la recompensa (elogios, alimento, juego y clickers); (b) basados en el castigo (denegación de contacto social, castigo vocal, remoción de estímulos placenteros, eventos repugnantes para el animal, cadena de estrangulamiento, echar agua en el rostro del perro o golpeteos en la oreja) y (c) otros, como usar correas largas, ignorar los errores, fortaleciendo la práctica y la paciencia.

Posteriormente, los hallazgos reportados por Rooney y Cowan (20II) confirmaron que los métodos de refuerzo basados en el castigo se asocian negativamente con un comportamiento social relajado del animal (Rho de Spearman $=-0,37 \mathrm{I}, n=5 \mathrm{I}$, $p=0,007)$ y con la capacidad del perro para ejecutar nuevas tareas de entrenamiento (Rho de Spearman = $-0,278, n=53, p=0,044)$, mientras que los refuerzos positivos se correlacionan positivamente con la habilidad del canino para aprender (Rho de Spearman $=0,308, n=53, p=0,025)$. Por otra parte, el castigo físico mostró asociación con una menor interacción durante el juego (U de Mann-Whitney = 187.5, $n=53$, $p=0.015)$ y estaban menos dispuestos a interactuar con el entrenador, en comparación con los perros a los que nunca se les ha sometido a esta clase de refuerzo.

Con base en lo planteado hasta el momento por los diversos autores, resulta evidente que la comprensión del alcance y efecto que tienen los diferentes tipos de refuerzo que se aplican en el proceso de entrenamiento, más allá del dispositivo que se esté empleando para que el animal logre el comportamiento deseado, es un factor crítico para el éxito en el aprendizaje de los caninos. En este sentido, se expone una revisión de los dispositivos empleados y algunos de los efectos más significativos en el comportamiento de perros dedicados a labores de seguridad y defensa. Así mismo, en la revisión se encuentran avances interesantes en la construcción de dispositivos destinados al adiestramiento canino, entre estos el desarrollado por Majikes et al. (20l7), quienes presentan un sistema semiautónomo, el cual sirve para controlar los estímulos mediante la combinación de elementos de hardware, software, audio y ayudas visuales; con capacidad para entrenar de manera autónoma al animal para que ejecute un comportamiento específico cuando se le dé la instrucción. Para lograrlo, se apoyan en el condicionamiento operante utilizando la luz y el sonido como estímulos discriminativos, contribuyendo de forma similar a la que realiza un entrenador profesional.
Según lo señalado por Majikes et al. (2017), los perros dedicados a labores de búsqueda y rescate (SAR, por sus siglas en inglés) cuentan con capacidades cognitivas, visuales, auditivas, olfativas y asociadas con la supervivencia que son difíciles de reemplazar por sistemas robóticos, demostrándose un rendimiento superior en muchos casos. No obstante, en las actividades de búsqueda existen situaciones en que el canino debe actuar de manera autónoma, con el fin de cubrir mayores áreas, dado que con el manejador podría encontrarse limitado en su movilidad, por lo tanto, la coordinación entre socorristas, adiestradores y animal es fundamental para lograr el objetivo.

Entre las principales variables que se deben supervisar en todo momento se encuentran la seguridad del terreno, la lectura del lenguaje corporal del perro, la evaluación continua de la ansiedad y el "golpe de calor", así como otras señales de su salud que lo pongan en una situación de riesgo. Por tal motivo Majikes et al. (2017) presentan un dispositivo cibernético para alcanzar mayor eficiencia en el entrenamiento de perros SAR. Así mismo, existen dispositivos que emplean la Unidad de Medición Inercial (IMU, por sus siglas en ingles), además de sensores fisiológicos que miden el ritmo cardíaco a través de electrocardiograma (ECG), sensor de fotopletismo (PPG) para medir aspectos relacionados con la respiración, controles de vibración, antena de Wireless Fidelity - WiFi (fidelidad inalámbrica) para comunicación, permitiendo un monitoreo en tiempo real y constante de la actividad del canino. Para ello, Majikes et al. (2017) diseñaron un algoritmo computacional que recopila e interpreta datos de los sensores, indicando la información significativa sobre cambios en el comportamiento del perro y ayudando a la mejora del entrenamiento, por ello, logra acelerar el aprendizaje, reducir costos en el proceso formativo e incrementar la disponibilidad y puesta en acción de estos valiosos recursos animales para labores de seguridad y defensa.

En cuanto a la utilidad, el dispositivo desarrollado por Majikes et al. (20l7) permite monitorear el canino con las IMU utilizando computadores que tienen la capacidad de detectar y corregir las malas posturas o comportamientos en tiempo real; así mismo, el ECG y el PPG ayudan a observar los signos vitales; por otro lado, facilitan la comunicación háptica (tacto) y aural (oído) empleando un sistema de audio y vibración controlado las órdenes de manera remota incluso si el animal no se encuentra en el rango de visión; además, el algoritmo permite recompensar a través de los dispensadores de tratamiento operados (DTO). Por último, los sensores ambientales proporcional datos 
relevantes sobre peligros potenciales a los que se puede enfrentar el perro de trabajo, aportando valiosa información para prevenir situaciones de riesgo.

En esta misma línea, existen los denominados collares de shock electrónicos, que son dispositivos colocados alrededor del cuello del animal para producir descargas de voltaje seguras o vibración para entrenar en comportamientos específicos (Coleman \& Murray, 2000). Sin embargo, Polsky (1994) había mencionado la diversidad de dispositivos clasificándolos en tres categorías principales: (a) control remoto con operación manual el cual se activa pulsando un botón; (b) collares antiladrido, que se activan cuando el perro ladra y (c) collares de cerco invisible, que son activados por una señal de radio emitida por un cable oculto bajo tierra.

Bajo estos lineamientos, Coleman y Murray (2000) investigaron la influencia de la utilización de estos dispositivos, tomando como referencia la

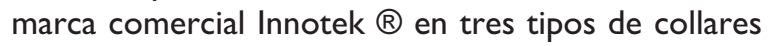
antiladrido, sistema de contención y collar de entrenamiento, encontrando que, si el entrenador sigue las recomendaciones sugeridas por el diseñador del aparato, es posible emplearlo como modificador eficaz de comportamientos problemáticos en los perros. Así mismo, Coleman y Murray (2000) reportan que no se observaron efectos adversos importantes en el animal por la utilización de estos collares; no obstante, consideran que sus hallazgos no pueden ser generalizados a toda la gama de dispositivos que se encuentran en el mercado, dado que es probable que elementos como técnicas de entrenamiento y refuerzo tengan una influencia significativa desconocida.

En relación con el entrenamiento mediante collares electrónicos, Blackwell et al. (20l2) indican que el uso ha sido controvertido en la literatura, por ello investigaron en una muestra por conveniencia de propietarios caninos en Inglaterra los factores de riesgo y percepción de los entrenadores respecto a estos dispositivos, y hallaron que solamente el 3,3\% de estos utilizaba collares de activación a distancia. Además, entre los factores de uso, los anteriores autores encontraron que son útiles para controlar comportamientos no deseados, como persecuciones espontáneas a vehículos, personas, otros animales, entre otros, así como la atención a llamados de regreso, ladridos, agresiones. No obstante, la percepción de los propietarios de perros en relación con el éxito esperado del método de entrenamiento varió significativamente entre los sistemas de refuerzo por recompensa y los collares electrónicos, siendo los primeros los que tuvieron una mejor evaluación respecto a los segundos.
Desde esta misma perspectiva, Salgirli et al. (2012) compararon los efectos en el aprendizaje y estrés canino que originan tres diferentes métodos de entrenamiento, entre estos los collares (electrónico y púas/compresión) y señales para abandono (quitting signal) en 42 perros policía; con ello se evaluaron aspectos como la concentración de cortisol en la saliva, reacciones y comportamientos, y se halló que el collar indujo menor cantidad de estrés y contribuyó mejor al aprendizaje comparado con los otros métodos, sobre todo con las señales, las cuales particularmente generaban altos niveles de cortisol. En concreto, Salgrili et al. (20I2) indicaron que el collar electrónico corrigió el comportamiento del 93\% de los caninos muestreados, mientras que los de púas lograron un cambio en el $76 \%$ y el uso de señales bajó al 7\% de efectividad, por lo que hubo evidencia estadísticamente significativa para confirmar que entre los collares (electrónico y púas) no hubo diferencias ( $p=0.16)$; sin embargo, sí se observó con respecto al uso de las señales $(p=0.00 \mathrm{I})$.

En relación con el comportamiento, se encontró que el collar electrónico obtuvo la máxima posición de la oreja hacia atrás al ser corregido, en contraste con el resto de dispositivos, por su parte, el indicador de la posición de cola en descenso no varió significativamente en ningún caso $(p=0.165)$, así mismo, el $4,8 \%$ de los perros mostró una bajada extrema en la postura corporal cuando se usó el collar de púas, mientras que esta reacción no surgió con el collar electrónico (Salgrili et al., 20I2). En definitiva, los niveles de cortisol más elevados se hallaron en perros cuando se usaron las señales de abandono (4l\%), seguido de collar electrónico (35\%) y collar de púas (24\%), concluyéndose que el collar electrónico presenta una mejor respuesta al aprendizaje con menos estrés para cesar el comportamiento no deseado; no obstante, el conocimiento, capacidad y habilidad del entrenador es un factor no controlado que debe tomarse en cuenta en futuros estudios.

Por su parte, Lines et al. (2013) realizaron una revisión de 2 I collares electrónicos comerciales utilizados en el Reino Unido (UK) debido a que existen muchos tipos de dispositivo pero pocos estudios comparaban las características técnicas en cuanto a la impedancia eléctrica, la cual se encontró que en condiciones húmedas fue de $10 \mathrm{k} \Omega$ y secas $640 \mathrm{k} \Omega$; además, se encontró que los estímulos generaban pulsos cortos de alto voltaje con diferencias significativas entre aspectos como picos, número de pulsos y duración, y se demostró un amplio rango en la fuerza de los estímulos de cada uno de los collares, así como la variación significativa entre los estímulos momentáneos y continuos. 
En relación con las características del collar, Lines et al. (20/3) mencionan que en promedio estas unidades poseen un largo entre 50 a $70 \mathrm{~mm}$ con 30 a $40 \mathrm{~mm}$ de ancho con la ubicación de dos sondas eléctricas que hacen contacto con la garganta del animal separadas entre sí a 30 a $50 \mathrm{~mm}, 10$ a 15 $\mathrm{mm}$ de largo y $5 \mathrm{~mm}$ de diámetro liso y redondo. No obstante, la evidencia demuestra que existen diferencias significativas en los estímulos según el tipo de collar que se utilice, por lo que los picos de tensión empleados varían con la resistencia del perro y suponen una falencia importante de estos dispositivos. Así mismo, se conoce que la impedancia del canino cambia en función de los estímulos y está influenciada por condiciones ambientales como estar seco o mojado.

Por lo tanto, en términos objetivos Lines et al. (2013) sugieren la construcción de algoritmos de validación de puntuación, con el fin de comparar los distintos collares en cuanto a su desempeño. Es importante mencionar que, a pesar de la pertinencia de la investigación realizada por Lines et al. (20/3), la Asociación de Fabricantes de Collares Electrónicos (ECMA, por sus siglas en inglés) reconoce que la literatura aun no recoge los avances logrados por esta organización y las mejoras realizadas a los dispositivos en los últimos años, sobre todo en aspectos relacionados con las indicaciones dirigidas al consumidor sobre la fuerza de la estimulación eléctrica y el uso adecuado, así que considera impulsar un mayor número de estudios en esta área, con el fin de contribuir con el conocimiento profundo de estas herramientas de entrenamiento (Riepl, 20I3).

Con base en lo dispuesto por la declaración de la European Society of Veterinary Clinical Ethology (ESCVCE) sobre el uso los collares electrónicos, estos puedes ser empleados como castigo positivo, si es utilizado luego del comportamiento indeseado - refuerzo negativo, manteniéndose hasta que se modifica la conducta y se muestra el comportamiento requerido; así mismo, han recopilado, a través de la literatura científica, una seria de ventajas entre las que destacan: (a) control preciso de la intensidad; (b) bajo costo, el cual es un argumento poco fiable si se toma como premisa el bienestar del animal; (c) contribuyen a reforzar el comportamiento alterno deseado; (d) representan menor riesgo que otros tipos de dispositivos; (d) demostraron ser efectivos para cambiar comportamientos de manera efectiva en comparación con otras técnicas. Por otro lado, entre las desventajas se indican: (a) parámetros como intensidad, duración, tamaño de electrodo y grado de humedad del perro son difíciles de controlar, dado que no hay consenso para determinar qué configuración es la más adecuada según el tipo de animal que se está entrenando; (b) asociación errada con estímulos externos; (c) abuso por parte del entrenador; (d) riesgos psicológicos incluyendo estrés.

Por su lado, Masson et al. (2018) argumentan que aún es poca la evidencia empírica que demuestre justificadamente la efectividad del uso de collares electrónicos, por lo que consideran que estos no deben ser usados, manifestando que existen métodos más eficientes de capacitación con menores riesgos; por ello, algunos países europeos, atendiendo a las recomendaciones de organismos internacionales como la ESCVCE, promueven legislaciones para evitar el uso, distribución y promoción, más allá de sus beneficios $y$ efectos colaterales en el animal, es un recurso que se encuentra en discusión actualmente. Además, Schalke y Hackbarth (2008) señalan que los factores que se analizan en la literatura están relacionados con cuestiones éticas y la pertinencia de usar el castigo más apropiado, por lo que sugieren que, desde el punto de vista científico, lo que se debe tomar en cuenta son las teorías de aprendizaje de los perros, así como las diferentes formas del condicionamiento, la manera en que hace la asociación el animal y adecuar correctamente la mejor forma de refuerzo y castigo.

El empleo de collares electrónicos para entrenamiento canino es amplio y versátil según las labores para las que se requiera, por ejemplo, Nguyen et al. (20I5) expone que el denominado Space and Naval Warfare Systems Center Pacific (SPAWAR), actualmente Naval Information Wafare Center Pacific (NIWC) y responsable de proporcionar soluciones a la armada de los Estados Unidos mediante actividades de investigación y desarrollo, diseñó un prototipo de collar que ayuda al canino a rastrear automáticamente explosivos, monitoreando en tiempo real el comportamiento del animal registrando sus datos vitales, así como aspectos relacionados con el tiempo de detección, capacidad de discriminación, recorrido; todo ello mediante el uso de sensores y cámaras especializadas.

Mediante el software del collar electrónico es posible rastrear la posición del perro y cómo hace su búsqueda, ayudando a identificar el comportamiento que señala la detección del explosivo; por su parte, la cámara registra condiciones ambientales que aportan más información a los entrenadores. Así mismo, el Global Positioning System (GPS) almacena la ruta seguida por el animal en un archivo con extensión XML, todos estos componentes contribuyen a determinar en qué momento preciso se encuentra en posición 
de detección. Complementariamente, se emplean técnicas estadísticas de clasificación, concretamente el algoritmo k-means a partir de los datos recogidos por el collar, con el fin de construir un modelo para proyectar tres aspectos: (a) capacidad de detección mediante cambios en la aceleración; (b) labores de búsqueda caracterizados por cambios normales en la aceleración y (c) movimientos bruscos denotados por alteraciones importantes en la aceleración. Además de los collares electrónicos existen otras herramientas, como el clicker, que consiste en la utilización de estímulos sonoros para modificar el comportamiento del perro, por ello, en los últimos años ha sido amplio el estudio de este tipo de dispositivos. Al respecto, Kaplan et al. (2002) propusieron la combinación de técnicas de entrenamiento canino para mejorar la interacción entre el hombre y robots, con el objetivo de crear un modelo para enseñar acciones complejas a un robot autónomo fundamentado en el uso de clicker.

Con este método Kaplan et al. (2002) pretenden contribuir a la mejora del proceso de entrenamiento de los dueños de perros de una forma segura para los animales, dado que al revisar la literatura encontraron que las técnicas para enseñar acciones en muchos casos se cometen numerosos errores que terminan por confundir e influenciar negativamente el comportamiento del canino; entre las más comunes se encuentran: (a) el modelado, que consiste en la manipulación física del animal para lograr la postura; (b) la atracción (luring), similar al modelado pero sin entrar en contacto con el perro, solo se usa la guía del entrenador para alcanzar la posición deseada con la mediación de juguetes o golosinas; (c) la captura (capturing) se basa en la recompensa cuando el canino realiza la postura de manera espontánea, por ello, forzar no es una premisa en este método; (d) la imitación, la cual es una técnica compleja no apta para todos los animales; $y$ (e) el uso de clicker, incluso mostrándose como un instrumento susceptible de ser empleado para modelar el comportamiento en robots de entrenamiento.

De acuerdo con Mehrkam y Wynne (20l4), entre los métodos de adiestramiento canino más populares se encuentran el refuerzo negativo, el método Koehler (1962), la formación basada en la relación social, la dominación y el uso de clicker como estímulo sonoro, todo ello fundamentado en modelos de aprendizaje que emplean el condicionamiento clásico, operante y no asociativo. Es importante mencionar que este dispositivo fue introducido en la industria del entrenamiento canino a mediados de los años ochenta por Pryor (2019) a través de su obra Don't Shoot the Dog; teóricamente, el clicker consiste en una caja pequeña normalmente de plástico que mediante un circuito electrónico genera un sonido característico y único al ser pulsado, este hecho produce en el animal la expectativa del comportamiento deseado donde se recibirá la recompensa, convirtiéndose este último en el estímulo incondicional, mientras que el clic será el estímulo condicional, lo que se enmarca dentro del paradigma clásico del proceso de aprendizaje Pavloviano (Mehrkam \& Wynne, 20I4).

A pesar del amplio uso de estos dispositivos, la revisión de literatura muestra que Smith y David (2008) reportan pocos estudios sobre la eficacia del entrenamiento con clicker en caninos; por tal motivo, analizan la eficacia de este instrumento de forma empírica enseñando a 35 basenjis a tocar un cono naranja con la nariz, estableciendo tres criterios de experimentación en diferentes ensayos para contrastar los avances, a saber: (a) ensayos de entrenamiento donde se dió refuerzo alimenticio y clic sonoro; (b) refuerzo variable (alimento y clic) para el fortalecimiento de la conducta de tocar el cono con la nariz y (c) ensayos de extensión, que consisten en retener alimento a los grupos pero reforzando con el clic la conducta.

Lo anterior permitió demostrar que el grupo control (sin clicker) y el experimental (con uso de clicker) no mostraron diferencias estadísticamente significativas en cuanto al número de ensayos o tiempo para cumplir los criterios de entrenamiento; esto sugiere que el clicker tiende a prolongar el comportamiento en ausencia de un refuerzo primario, sin embargo, no reduce el tiempo de entrenamiento de una tarea operante cuando se retrasa dicho refuerzo; así mismo, es útil para mantener comportamientos aprendidos, si el refuerzo primario no se encuentra $\circ$ es difícil entregarlo de forma práctica, incrementando la resistencia a la extinción de la conducta (Smith \& David, 2008). De forma similar, Chiandetti et al. (2016) verificaron la eficacia de entrenamiento con clicker evaluando el comportamiento en tres condiciones distintas: (a) con reforzador primario se presentaba asociado al sonido del dispositivo; (b) una palabra, condición que está ausente en trabajos previos sobre clickers y (c) solo sin estímulo. Los hallazgos confirman que los perros aprendían por igual, sin diferencias significativas asociadas al refuerzo acústico secundario, en comparación con cuando se presentaba el refuerzo primario únicamente.

Sin embargo, Feng et al. (2016) reseñan que los estudios sobre la eficacia de clickers para el aprendizaje de los perros son limitados. Además, sugieren que son utilizados comúnmente como reforzadores 
secundarios, siendo más efectivos cuando vienen acompañados por actividades que liberen dopamina en el animal; en función de lo anterior, indican que es importante entender cómo funcionan estos dispositivos para seguir las mejores prácticas de su uso. Los mismos autores señalan también que los experimentos científicos que respaldan la contribución del clicker al aprendizaje son pocos: en concreto, de cinco investigaciones que comparaban un grupo de perros que recibían señal y alimento contra un grupo de solo alimento, apenas uno encontró que los animales con ambos refuerzos aprendían más rápido.

Con base en lo anterior, Feng et al. (2016) contrastaron tres métodos que denominan: (a) hipótesis de refuerzo (Skinner, 1938), en el que el clicker se convierte en un refuerzo secundario; (b) hipótesis de marcado (Lieberman et al., 1979), que sugiere la exposición de señales presentadas en un contexto del comportamiento objetivo para facilitar el aprendizaje; y (c) la hipótesis de vínculo de Kaplan y Hearst (1982), la cual indica que las señales mostradas entre el comportamiento objetivo y los actos de recompensa logran crear una conexión o puente entre dicho comportamiento y la consecuencia. A partir de las pruebas realizadas por Feng et al. (20l6), se determinó que los clickers funcionan como reforzadores condicionados, siempre y cuando su uso esté emparejado con un refuerzo primario para que logre convertirse en una señal predictora; igualmente, sirven como dispositivos para marcar conductas; no obstante, es necesario desarrollar más estudios que permitan analizar factores adicionales como el número de emparejamientos entre señales y refuerzos primarios requeridos para enseñar tareas nuevas, calidad e intensidad de la señal -por ejemplo, si sonidos cortos y agudos son más eficaces para marcar o tonos extensos logran resultados diferentes en establecer vínculos.

La evidencia empírica sugiere que el clicker puede ser empleado para enseñar tareas de discriminación visual en los caninos. En este caso, Autier et al. (2013) experimentaron con nueve perros para entrenarlos en labores de reconocimiento de otras especies caninas mediante la visualización de dos imágenes en pantallas de ordenadores, y se halló que los perros cuentan con capacidades para discriminar especies aun cuando los fenotipos son amplios y variados; además, se demostró en parte la utilidad del clicker como método de entrenamiento. En este orden de ideas, Strychalski et al. (20/5) manifestaron la escasez de estudios relacionados con el análisis del comportamiento de las razas ante el uso de clicker; por ello, observaron las reacciones de boxer, chow chow y Yorkshire terrier frente a este dispositivo, hallando que los boxer no se adaptaban correctamente a los primeros intentos respecto a los Yorkshire terrier $(p<0.05)$, mientras que los chow chow lograban la conducta más efectivamente al inicio, pero requerían mayores sesiones para mantenerla, en comparación con el resto; por lo tanto, detectaron que, a pesar de los resultados, es posible que los chow chow puedan tener éxito en este tipo de tareas si se emplea correctamente el uso del clicker.

Por otro lado, Feng et al. (2017), a través de la revisión de la literatura y entrevistas a expertos, encontraron que el entrenamiento con clicker cuenta con dos enfoques: el primero lo identifica como una simple técnica, mientras que el segundo lo asocia con un paradigma filosófico de adiestramiento mucho más amplio. Además, la experiencia de profesionales indica que este dispositivo se debe usar cuando se entrenan nuevos comportamientos, pero no como recompensa cuando el animal cuente con comportamientos conocidos; aunado a lo anterior, aún no hay acuerdo científico sobre la eficacia relativa de los clicker frente a otro tipo de señales sonoras (Feng et al., 2017).

\section{Patentes asociadas con dispositivos de entrenamiento canino}

Hasta el momento, es evidente la utilidad de los dispositivos para el entrenamiento canino, pues permiten el aprendizaje de comportamientos deseados; no obstante, la investigación científica sobre su eficacia y condiciones correctas de empleo parece ser escasa. Por otra parte, en los últimos años ha venido creciendo considerablemente el número de patentes en el sector de adiestramiento de perros. Al revisar en algunas bases de datos sobre invenciones asociadas con dispositivos para adestramiento, se encontraron los resultados presentados en la tabla 3.

\section{Tabla 3}

\section{Ecuaciones para la búsqueda de patentes}

\begin{tabular}{|c|c|c|}
\hline Buscador & Ecuación de búsqueda & Resultados \\
\hline Google Patents & a) $(($ TI = (DOG TRAINING)) type:PATENT & 1.173 \\
\hline Espacenet & b) ((TI = (DOG TRAINING BOX)) type:PATENT & 12 \\
\hline PatentScope & c) ta all “dog" AND ta all “training” AND ta all "device" & 350 \\
\hline
\end{tabular}

Fuente: elaboración propia 
Tabla 4

Resumen de patentes relacionadas con cajas de entrenamiento

\begin{tabular}{|c|c|c|c|}
\hline ID & Título & Año & Link acceso \\
\hline CN-20889|437-U & $\begin{array}{l}\text { A kind of dog smell box, smell tank, training } \\
\text { tank and smell search platform }\end{array}$ & 2018 & https://is.gd/Qm2 $2 u$ \\
\hline CN-205I6I488-U & Remote control search dog training box & 2015 & https://clck.ru/Rihsk \\
\hline CN-205I61489-U & Manually roll formula search dog training box & 2015 & https://clck.ru/RihtR \\
\hline CN-205I6I487-U & Open search dog training box by oneself & 2015 & https://clck.ru/Rihuz \\
\hline ES-II36009-U & $\begin{array}{l}\text { Dog training discoloration box (Machine-translation } \\
\text { by Google Translate, not legally binding) }\end{array}$ & 2014 & https://is.gd/odK8DI \\
\hline CN-202565946-U & Multi-functional smell training box for police dogs & 2012 & https://clck.ru/Rihwc \\
\hline DE-02010016131-UI & $\begin{array}{l}\text { Training box for the passive display of objects } \\
\text { (fragrances / objects) for special and search dogs }\end{array}$ & 2010 & https://clck.ru/RihxP \\
\hline CN-20I797843-U & Training box for pet to search & 2010 & https://clck.ru/Rii2c \\
\hline NL-1036I I8-C2 & $\begin{array}{l}\text { Portable box for obedience training of dogs, has } \\
\text { button and bell or lamp, which allows dogs to } \\
\text { indicate that they have completed assigned task }\end{array}$ & 2008 & https://clck.ru/Rii3Z \\
\hline FR-2854028-BI & $\begin{array}{l}\text { Draft box for dog training, on the principle of torsion } \\
\text { spring and magnetic lock spring arm, intended for } \\
\text { the bird lacher introduced by opening a trapper }\end{array}$ & 2003 & https://clck.ru/Rii6G \\
\hline SE-525I29-C2 & $\begin{array}{l}\text { Animal training device, especially for dogs, } \\
\text { comprises box designed to dispense reward } \\
\text { when object such as block is placed inside it }\end{array}$ & 2002 & https://clck.ru/Rii75 \\
\hline
\end{tabular}

Fuente: Google Patents, 2020.

En concreto, al analizar los 1.173 resultados de Google Patents, se encontró que el $46,2 \%$ de los dispositivos diseñados se concentra en la categoría AOIK, que, según la Clasificación Internacional de Patentes (WIPO, por sus siglas en inglés), engloba una amplia cantidad de elementos asociados con el entrenamiento animal. Así mismo, el mayor número de patentes $(I, 7 \%)$ ha sido presentado por la Base de Entrenamiento de Perros de la Policía de Harbin, la Fuerza de Policía Armada Popular y Cuerpo de Guardia de Fronteras de la Provincia de Heilongjiang, todas estas ubicadas en China.

En el caso de los resultados arrojados por Patent Scope, el $39 \%(n=138)$ de las patentes proviene de China y el $33 \%(n=117)$ corresponde a Estados Unidos, con lo cual estos dos países se posicionan como los de mayor producción e innovación en el ámbito de los dispositivos de entrenamiento canino, con una concentración del $78 \%(n=274)$ en la categoría A0IK y del 9,4\% $(n=33)$ en A63B. Por su parte, el mayor número de patentes se registra entre los años 2017, 2018 y 2019 , con 34, 47 y 5I, invenciones respectivamente. Por otro lado, al indagar en las patentes específicas para cajas de entrenamiento señaladas por la ecuación de búsqueda (b), se encontró que su finalidad es apoyar el entrenamiento de perros en labores de búsqueda, discriminación y detección; seguidamente, la tabla 4 muestra las características principales de estas patentes.

En función de la producción de patentes relacionadas con dispositivos de entrenamiento tipo cajas, se observa la necesidad de construir prototipos que contribuyan al aprendizaje canino, incorporando e integrando nuevos elementos que faciliten el adiestramiento efectivo. Por tal razón, desde la EGAC se considera pertinente aportar al mercado ideas para el desarrollo de un dispositivo específico para el entrenamiento de perros de trabajo. Con base en la experiencia y utilización de cajas de madera se han generado ideas para el diseño y desarrollo de un dispositivo patentable que combine diferentes estímulos atendiendo a las siguientes características: (a) Estabilidad: mediante forma hexagonal se puede lograr un mejor ajuste del dispositivo al suelo, con ello sería posible evitar que el canino mueva la caja y provocar el derrame de las sustancias; (b) Independencia de olores: conductos independientes para crear un proceso de discriminación más efectivo; y (c) Estímulo auditivo: la incorporación de sonidos como refuerzo secundario para el aprendizaje puede contribuir considerablemente en el logro del comportamiento deseado.

Por lo anterior, el adiestramiento canino es un proceso complejo en el que intervienen numerosas 
variables, entre estas el sistema de refuerzo utilizado, el tipo de condicionamiento más adecuado y los dispositivos empleados para lograr la conducta deseada, sobre todo en aquellos animales previstos para realizar actividades de seguridad y defensa. $\mathrm{Si}$ bien el objetivo de esta revisión no es discutir el comportamiento asociado a la respuesta de los perros frente a los dispositivos, es de aclarar que en el contexto de las fuerzas policiales y militares, Haverbeke et al. (2008) confirman que son pocos los estudios que analizan los métodos y dispositivos utilizados en el adiestramiento canino con fines de seguridad y defensa; además, sugieren que el rendimiento del perro está directamente asociado con el sistema de refuerzo y la capacidad de concentración del animal, por lo que se infiere que las herramientas que se empleen en este proceso (clickers, cajas, collares, entre otros), así como la relación entre el binomio (adiestrador-canino), forman parte integral de la respuesta ante los estímulos y el logro de las conductas deseadas, que mejorarían en gran medida la sinergia entre los dispositivos electrónicos y los sistemas de refuerzo físico, y probablemente aumentarían el rendimiento del perro de trabajo en escenarios reales.

En este orden de ideas, Lazarowski et al. (2020a) argumentan que la detección de explosivos por medio de perros es uno de los métodos más efectivos en la actualidad, así como identificación de narcóticos, fauna silvestre y diagnóstico médico (Lazarowskyi et al., 2020b), como por ejemplo la presencia de covid-19 en pacientes (Jendrny et al., 2020), entrenamientos que emplean herramientas tecnológicas, especialmente para discriminar el olor, siendo uno de los focos de atención prioritario para certificar los perros detectores.

La escasez de estudios científicos y protocolos normalizados en el sector de entrenamiento canino crea nuevos retos y dificultades al momento de tomar decisiones asociadas con la capacidad, vulnerabilidad y riesgo, donde los perros detectores añaden dificultad e inexactitudes a la hora del proceso de entrenamiento (Lazarowskyi et al., 2020b). Así mismo, el refuerzo empleado para fijar la conducta en el perro resulta crítico para restringir los comportamientos no deseados en el animal (Mclntire, 1968; Fonberg \& Kostarczyk, 1980), dado que está demostrado que los caninos son sensibles a las señales del ser humano. Sin embargo, se requieren más estudios que analicen en profundidad el efecto de las latencias en las entregas de los refuerzos primarios y secundarios (Browne et al., 20ll), especialmente en el momento en que se asocia una sustancia durante el entrenamiento.
En este orden de ideas, Browne et al. (2013) afirman que existe una relación entre el retraso del refuerzo y el aprendizaje, pero esta no es constante en todos los perros, es decir, la oportunidad en la que se entrega la recompensa resulta ser una variable condicionada en el adiestramiento. En concordancia con lo anterior, Mercier et al. (1988) ya habían sostenido esta hipótesis, confirmando que el refuerzo diferencial de bajas tasas de respuesta (DRL, por sus siglas en inglés) genera patrones de comportamiento colateral, es decir, actividades motoras que usa el perro para compensar mecanismos inhibidores en el contexto de un condicionamiento operante, por ello, pasa a ser una simple consecuencia creada por un estado de inhibición en la recompensa.

En cuanto al comportamiento, Brady et al. (20l8) afirman que es generalizada la escasez de información asociada con la fiabilidad y validez de las medidas para evaluar este aspecto en los perros; igualmente, identificaron incoherencias en la terminología, parámetros experimentales e índices de éxito. En adición con lo anterior, Jamieson et al. (2018) señalan que cuando un canino es manejado por una persona desconocida para el animal, existe más probabilidad de tener comportamientos indeseados causados por estrés, lo cual aumenta la distracción y afecta negativamente el rendimiento en la detección, e incluye una nueva variable de influencia en el perro de trabajo. Al ser el perro un animal social, su conducta puede verse alterada por elementos del entorno y la relación con el ser humano, independientemente del dispositivo que se esté utilizando para el aprendizaje (Herwijnen et al., 20 I8); en este sentido, deben tenerse en consideración aspectos como la experiencia previa del canino en tareas de entrenamiento (Marshall et al., 2009; Marshall et al., 2016), el método de refuerzo (Hiby et al., 2004; Rooney \& Cowan, 20 I I) y el comportamiento del propietario o entrenador (Arhant et al., 20l0).

Por su parte, en la literatura se ha revisado en profundidad el proceso de aprendizaje canino como un aspecto de interés (Tolman, 1925; Mackintosh, 1974; Dickinson, 1980); (Castro \& Wasserman, 2010); (Pearce, 20I3); no obstante, los dispositivos de entrenamiento como apoyo para lograr conductas deseadas han sido controvertidos, entre estos el collar electrónico (Polsky, 1994; Hughes, 2003); (Schalke et al., 2007), que genera estrés y conductas no deseadas (Schilder \& Van der Borg, 2004); además, las características técnicas difieren según marcas y modelos, lo que hace aún más difícil el proceso de entrenamiento (Lines et al., 2013; Riepl, 2013). En contraposición, algunos autores, como Salgrili 
et al. (2012), defienden su utilidad afirmando que contribuyen significativamente a la corrección de comportamientos de forma más efectiva que otros dispositivos similares.

Con respecto al clicker, Feng et al. (2018) afirman que es un refuerzo secundario que funciona mejor cuando es utilizado con una recompensa primaria como la comida; así mismo, Cimarelli et al. (2020) señalan que este dispositivo se asocia con el refuerzo positivo y manifiestan que en la literatura aún no existe un consenso sobre la efectividad cuando se utiliza únicamente el clicker o cuando viene acompañado de alimento; además, concluyen que la recompensa parcial no mejora la eficiencia del entrenamiento. Por el contrario, Dorey et al. (2020) indican que no hay evidencia empírica que confirme que la adición de un clicker mejore el rendimiento del entrenamiento realizado con refuerzo primario alimenticio o solamente con sonido, por ello es necesario realizar más estudios para determinar los beneficios del clicker (Dorey \& Cox, 20l8).

Cabe señalar que el incremento del interés en la comunidad científica para el desarrollo de estudios sobre la eficiencia, efectividad e impacto de los diferentes dispositivos ha venido consolidándose en los últimos años. En virtud de lo anterior, es necesario desarrollar prototipos que permitan integrar estímulos sonoros utilizados como refuerzo secundario, así como recompensas primarias, las cuales pueden contribuir a mejorar el proceso de entrenamiento en el marco de las teorías de aprendizaje de los perros. El diseño y la construcción de dispositivos que cumplan con este propósito podrían constituir un aporte significativo a la generación de conocimiento en el área del adiestramiento canino en el contexto de las labores de seguridad y defensa nacional. Aunque existe un interés creciente en investigar el impacto del entrenamiento sobre la cognición canina, pocos estudios exploran la efectividad de varios métodos de entrenamiento sobre ella (Silver et al., 2020); se requieren enfoques experimentales con pruebas que midan los encuentros sociales, el juego, los eventos inesperados y los escenarios relacionados durante la búsqueda, especialmente al utilizar instrumentos para el entrenamiento.

Los equipos caninos posteriores al entrenamiento son validados en el trabajo real operacional, configurando su capacidad de rendimiento; es ahí donde se expresa y se observa el resultado del entrenamiento, empleados como caninos detectores de sustancias ilícitas, el hallazgo del olor humano o las ciencias forenses, con un rendimiento superior al humano; sin embargo, no todos los perros de trabajo que ingresan a los programas académicos de entrenamiento tienen éxito, elevando los costos en los procesos de selección, adiestramiento, certificación y rendimiento. Así mismo, el número de animales disponibles que ingresan a dichos programas es limitado, debido a las exigencias sanitarias actuales y al "perfil zootécnico ideal en las razas existentes", que se diferencian de los perros de compañía. Entrenar un perro es una labor desafiante en un país como Colombia, donde se debe optimizar la eficiencia cuando se está entrenando, con una sinergia entre el manejador, su perro y el instructor, especialmente al utilizar las ayudas de entrenamiento y los dispositivos electrónicos, los cuales son empleados según cada contexto y requerimientos, siguiendo protocolos ajustados, estandarizados y con unas exigencias de calidad impartidos por los expertos entrenadores.

\section{Conclusiones}

En Colombia son pocos los artículos generados sobre los perros detectores y menos los que emplean dispositivos electrónicos o instrumentos para el entrenamiento; en Latinoamérica existen pocos artículos sobre el tema. En Estados Unidos e Inglaterra el número va en aumento, conformando grupos fuertes de investigación con la evaluación de un número importante de animales. Por lo anterior, existen oportunidades en las líneas de investigación de los perros detectores, especialmente los asociados a la seguridad y convivencia ciudadana, cuya función en Colombia la ejerce la Policía Nacional, anudado a las diversas especialidades del servicio del guía canino, las cuales dependen de las diferentes técnicas utilizadas para el adiestramiento, en las que se requieren protocolos y estándares validados, junto a un trabajo colaborativo interdisciplinar, y consensuar a los investigadores en estas líneas del conocimiento, según lo observado en la revisión de la literatura.

Debe existir un conocimiento sobre las teorías de aprendizaje del perro por parte del entrenador, con el fin de lograr las conductas deseadas. Además, durante los últimos veinte años el entendimiento científico y empírico del comportamiento animal ha crecido y se ha expandido rápidamente, al igual que la experiencia y los métodos de adiestramiento de los perros de trabajo. Por lo anterior, se espera que esta publicación inspire nuevas direcciones colaborativas entre investigadores y practicantes, con el objetivo de ampliar la información y las técnicas basadas en la evidencia, las cuales los futuros entrenadores puedan incorporar dentro de su experticia. La sinergia de 
los instrumentos para el entrenamiento y el vínculo entre manejador y canino deben ser comprendidos y analizados, posiblemente resultando en mejores prácticas al usar la tecnología, para obtener un impacto medible en el bienestar del binomio y la disponibilidad de las ayudas de entrenamiento durante el refuerzo positivo, de modo tal que sea posible mejorar en los perros el cumplimiento de los roles necesarios en nuestra sociedad.

Se confirma la falta de estudios empíricos relacionados con el impacto del uso de herramientas tecnológicas durante el entrenamiento, pues existe una amplia variedad de características técnicas, marcas y especificaciones que posiblemente no permiten medir su efectividad desde el punto de vista científico. Además, es crucial la normalización y estandarización de las variables que intervienen en el entrenamiento, con el fin de entender de una mejor manera el comportamiento y el conocimiento sobre los factores que predicen el rendimiento óptimo de los caninos.

Tanto el uso de clicker como collares electrónicos, arnés de control de estímulos, cajas y carruseles permiten un manejo y una evolución comportamentales, especialmente en estudios empíricos de los programas académicos que emplean binomios (guíacanino). Las diversas variables que se involucran en el proceso de entrenamiento son elementos que deben ser tomados en cuenta por el ser humano que dirige el aprendizaje. Finalmente, en el caso de las cajas de recompensa automática, se encontraron pocos estudios sobre su efectividad; sin embargo, vale señalar que es un dispositivo ampliamente utilizado en Colombia, el cual fija el foco de atención del perro sobre el elemento motivador, permitiendo caninos con un "drive" ideal para discriminar el olor blanco durante el entrenamiento. Lo anterior resalta que los procedimientos de refuerzo positivo pueden ser tan eficientes y efectivos como los collares electrónicos. Los procedimientos de refuerzo positivo pueden construir un vínculo humano-animal más fuerte y evitar los riesgos potenciales para el bienestar de las técnicas aversivas; por lo tanto, identificar métodos de entrenamiento para perros de trabajo que no solo sean altamente efectivos, sino que también promuevan el bienestar animal y el vínculo humano-animal es un futuro importante que se debe investigar.

Para concluir, el rol de los dispositivos en el entrenamiento de caninos dedicados a labores de seguridad y defensa es determinante para el aprendizaje de conductas que permitan a las autoridades identificar de manera efectiva actividades ilícitas que pongan en riesgo a la ciudadanía. Sin embargo, los avances y mejoras de los últimos años en este ámbito, demostrados por el número de patentes desarrolladas, presentan dificultades en torno a la medición científica del impacto en el servicio policial. Desde una perspectiva metodológica, el presente artículo examinó el papel de los dispositivos en el entrenamiento observando los efectos, limitaciones y consideraciones, determinando que el conocimiento del entrenador respecto a las teorías de aprendizaje canino, el contexto donde se desarrollan los ejercicios con el animal y las características técnicas de dichos dispositivos pueden generar diversos escenarios de entendimiento. Por tal motivo, es primordial impulsar un mayor número de investigaciones en este campo con el apoyo de instituciones y de expertos de varias disciplinas.

\section{Conflicto de intereses}

Los autores declaran que no existe ningún potencial conflicto de interés relacionado con el artículo. Además, este trabajo forma parte de una investigación amplia, derivada del proyecto "Tecnología sensorial y multimedia en el entrenamiento de caninos detectores de sustancias", realizado por los integrantes del grupo de investigación "Olfateando el conocimiento" de la Escuela de Guías y Adiestramiento Canino PONALESGAC, grupLAC: COL006435I, como estrategia para mejorar el entrenamiento de los caninos de trabajo de la Policía Nacional e impactar la seguridad urbana y rural colombiana. Los autores desean agradecer a la Policía Nacional de Colombia, Escuela de Guías y Adiestramiento Canino, al Research Center Working Dogs $®$ y a Luis A. Montenegro por la consultoría y asesoría.

\section{Referencias}

Arhant, C., Bubna-Littitz, H., Bartels, A., Futschik, A., \& Troxler, J. (20I0). Behaviour of smaller and larger dogs: Effects of training methods, inconsistency of owner behaviour and level of engagement in activities with the dog. Applied Animal Behaviour Science, I 23(3-4), I 3 |- I 42.

Autier-Dérian, D., Deputte, B. L., Chalvet-Monfray, K., Coulon, M., \& Mounier, L. (20I3). Visual discrimination of species in dogs (Canis familiaris). Animal Cognition, I6(4), 637-65I.

Beltrán, O. A. (2005). Revisiones sistemáticas de la literatura. Revista colombiana de gastroenterología, 20(1), 60-69. 
Bentosela, M., Barrera, G., Jakovcevic, A., Elgier, A. M., \& Mustaca, A. E. (2008). Effect of reinforcement, reinforcer omission and extinction on a communicative response in domestic dogs (Canis familiaris). Behavioural processes, 78(3), 464-469.

Blackman, D. E. (2017). Operant conditioning: an experimental analysis of behaviour. Routledge.

Blackwell, E. J., Bolster, C., Richards, G., Loftus, B. A., \& Casey, R. A. (20I2). The use of electronic collars for training domestic dogs: estimated prevalence, reasons and risk factors for use, and owner perceived success as compared to other training methods. BMC Veterinary Research, 8(I), 93.

Blackwell, E. J., Twells, C., Seawright, A., \& Casey, R. A. (2008). The relationship between training methods and the occurrence of behavior problems, as reported by owners, in a population of domestic dogs. Journal of Veterinary Behavior, 3(5), 207-2I7.

Brady, K., Cracknell, N., Zulch, H., \& Mills, D. S. (20I8). A systematic review of the reliability and validity of behavioural tests used to assess behavioural characteristics important in working dogs. Frontiers in veterinary science, $5,103$.

Browne, C. M., Starkey, N. J., Foster, T. M., \& McEwan, J. S. (20|3). Delayed reinforcement-does it affect learning. Journal of Veterinary Behavior: Clinical Applications and Research, 8(4), e37-e38.

Browne, C. M., Starkey, N. J., Foster, T. M., \& McEwan, J. S. (20I I). Timing of reinforcement during dog training. In 2nd Canine Science Forum: Clinical Applications and Research (Vol. 6, No. I, pp. 58-59).

Cáceres, J., \& Rojas-Guevara, J. (2020). II Congreso latinoamericano de comportamiento y bienestar animal (ISAE). Ponencia: La investigación criminal de los delitos sexuales a través de perros detectores de residuos biológicos. Relaciones actuales entre humanos y animales. Medellín, Colombia. Revista Facultad Nacional de Agronomía Medellín. (202I), 74 (Suplemento), SI69-I85.

Castro, L., \& Wasserman, E. A. (20I0). Animal learning. Wiley Interdisciplinary Reviews: Cognitive Science, I (I), 89-98.

Chiandetti, C., Avella, S., Fongaro, E., \& Cerri, F. (20|6). Can clicker training facilitate conditioning in dogs? Applied Animal Behaviour Science, 184, 109-1 16.

Cimarelli, G., Schoesswender, J., Vitiello, R., Huber, L., \& Virányi, Z. (2020). Partial rewarding during clicker training does not improve naïve dogs' learning speed and induces a pessimistic-like affective state. Animal Cognition, I- I3.

Coleman, T., \& Murray, R. (2000). Collar mounted electronic devices for behaviour modification in dogs. In Proceedings of the Urban Animal Management Conference, http://www.ava. com. au/UAM/proc00/murray.htm.

Córdoba-Parra, J., Rojas Guevara, J., Prada-Tiedemann, P., \& Bohórquez, G. (2020). II Congreso latinoamericano de comportamiento y bienestar animal (ISAE). Ponencia: Variables ambientales que afectan la interacción humano-animal: pieza clave para hallar desaparecidos con perros policía. Relaciones actuales entre humanos y animales. Medellín, Colombia.

Creswell, J. W., \& Poth, C. N. (2016). Qualitative inquiry and research design: Choosing among five approaches. Sage.

Deruyter, E., Nettles, K., Ochoa-Torres, M., Cristancho, O., Rojas-Guevara, J., Bohórquez, G., \& PradaTiedemann, P. (2020). What's that lingering smell? Evaluation of residual odor volatiles in Colombian territory. Texas Tech University Undergraduate Research Conference (URC). Institute for Forensic Science, Department of Environmental Toxicology, Texas Tech University. https://www.depts.ttu.edu/true/ urc/2020/poster-files/poster_deruyter.pdf.

Dickinson, A. (1980). Contemporary animal learning theory (Vol. I). CUP Archive.

Dorey, N. R., \& Cox, D. J. (2018). Function matters: a review of terminological differences in applied and basic clicker training research. PeerJ, 6, e562I.

Dorey, N. R., Blandina, A., \& Udell, M. A. (2020). Clicker training does not enhance learning in mixed-breed shelter puppies (Canis familiaris). Journal of Veterinary Behavior, 39, 57-63.

European Society of Veterinary Clinical Ethology (ESCVCE). (2020). Electronic Traning Device. http://www.esvce.org/wp-content/ uploads/20I 7/ I I/ESVCE-Position-Statemente-collar.pdf.

Feng, L. C., Howell, T. J., \& Bennettx., P. C. (2016). How clicker training works: comparing reinforcing, marking, and bridging hypotheses. Applied Animal Behaviour Science, I8I, 34-40.

Feng, L. C., Howell, T. J., \& Bennett, P. C. (2017). Comparing trainers' reports of clicker use to the use of clicker in applied research studies: Methodological differences may explain conflicting results.

Fonberg, E., \& Kostarczyk, E. (1980). Motivational role of social reinforcement in dog-man relations. Acta Neurobiologiae Experimentalis, 40, II 7. 
Gabrielsen, A. M. (2017). Training Technologies. Science, Gender and Dogs in the Age of Positive Dog Training. Nordic Journal of Science and Technology Studies, 5(I), 5-I6.

Goldkuhl, G. (20I2). Pragmatism vs interpretivism in qualitative information systems research. European journal of information systems, 2 I (2), |35- 46.

Haverbeke, A., Laporte, B., Depiereux, E., Giffroy, J. M., \& Diederich, C. (2008). Training methods of military dog handlers and their effects on the team's performances. Applied Animal Behaviour Science, I I 3( I-3), I I0- I 22.

Herwijnen, I. R. V., van der Borg, J. A., Naguib, M., \& Beerda, B. (20/8). Dog ownership satisfaction determinants in the owner-dog relationship and the dog's behaviour. Plos one, 13(9), e0204592.

Hiby, E. F., Rooney, N. J., \& Bradshaw, J. W. S. (2004). Dog training methods: their use, effectiveness and interaction with behaviour and welfare. Animal welfare-potters bar then wheathampstead-, I3(I), 63-70.

Higgins, J. P., Thomas, J., Chandler, J., Cumpston, M., Li, T., Page, M. J., \& Welch, V. A. (Eds.). (2019). Cochrane handbook for systematic reviews of interventions. John Wiley \& Sons.

Hughes, E. J. (2003). Electric shock collars and dog training. The Veterinary record, I53(19), 604.

Jamieson, L. T. J., Baxter, G. S., \& Murray, P. J. (20I8). You are not my handler! Impact of changing handlers on dogs' behaviours and detection performance. Animals, 8(I0), I76.

Jendrny, P., Schulz, C., Twele, F., Meller, S., von Köckritz-Blickwede, M., Osterhaus, A. D. M. E., ... \& Manns, M. P. (2020). Scent dog identification of samples from covid-19 patients-a pilot study. BMC infectious diseases, 20(I), I-7.

Kaplan, P. S., \& Hearst, E. (I 982). Bridging temporal gaps between CS and US in autoshaping: insertion of other stimuli before, during, and after CS. Journal of Experimental Psychology: Animal Behavior Processes, 8(2), 187.

Kaplan, F., Oudeyer, P. Y., Kubinyi, E., \& Miklósi, A. (2002). Robotic clicker training. Robotics and Autonomous Systems, 38(3-4), 197-206.

Kitchenham, B., Brereton, O. P., Budgen, D., Turner, M., Bailey, J., \& Linkman, S. (2009). Systematic literature reviews in software engineering-a systematic literature review. Information and software technology, 5 I (I), 7-I5.

Koehler, W. R. (1962). The Koehler method of dog training. Howell Books.

Lazarowski, L., Waggoner, L. P., Krichbaum, S., Singletary, M., Haney, P. S., Rogers, B., \& Angle, C. (2020a). Selecting dogs for explosives detection: Behavioral characteristics Frontiers in Veterinary Science, 7, 597.

Lazarowski, L., Krichbaum, S., DeGreeff, L. E., Simon, A., Singletary, M., Angle, C., \& Waggoner, L. P. (2020b). Methodological Considerations in Canine Olfactory Detection Research. Frontiers in Veterinary Science, 7.

Lieberman, D. A., Mclntosh, D. C., \& Thomas, G. V. (1979). Learning when reward is delayed: A marking hypothesis. Journal of Experimental psychology: Animal behavior processes, 5(3), 224.

Mackintosh, N. J. (1974). The psychology of animal learning. Academic Press.

Majikes, J. J., Yuschak, S., Walker, K., Brugarolas, R., Mealin, S., Foster, M., ... \& Roberts, D. L. (2017). Stimulus Control for Semiautonomous Computer Canine-Training. In Conference on Biomimetic and Biohybrid Systems (pp. 279-290). Springer, Cham.

Marshall-Pescini, S., Passalacqua, C., Barnard, S., Valsecchi, P., \& Prato-Previde, E. (2009). Agility and search and rescue training differently affect pet dogs' behaviour in sociocognitive tasks. Behavioural processes, $8 I(3)$, 4I6-422.

Marshall-Pescini, S., Frazzi, C., \& Valsecchi, P. (20I6). The effect of training and breed group on problem-solving behaviours in dogs. Animal Cognition, 19(3), 57|-579.

Masson, S., de la Vega, S., Gazzano, A., Mariti, C., Pereira, G. D. G., Halsberghe, C., ... \& Schoening, B. (20/8). Electronic training devices: Discussion on the pros and cons of their use in dogs as a basis for the position statement of the European Society of Veterinary Clinical Ethology. Journal of Veterinary Behavior, 25, 7I-75.

Mclntire, R. W., \& Colley, T. A. (1967). Social reinforcement in the dog. Psychological Reports, 20(3), 843-846.

Mclntire, R. W. (1968). Dog training, reinforcement, and behavior in unrestricted environments. American Psychologist, 23( I I), 830.

Mehrkam, L. R., \& Wynne, C. D. (20I4). Behavioral differences among breeds of domestic dogs (Canis lupus familiaris): Current status of the science. Applied Animal Behaviour Science, I 55, 12-27.

Mengist, W., Soromessa, T., \& Legese, G. (2020). Method for conducting systematic literature review and meta-analysis for environmental science research. MethodsX, 7, 100777.

Mercier, M., Chleide, E., \& Bruhwyler, J. (1988). Behaviour, using a differential reinforcement of response duration (drrd) schedule with external cues, in the dog. Psychologica Belgica, 28(2), I23-137. 
Nguyen, H. G., Nans, A., Talke, K., Candela, P., \& Everett, H. R. (20I5, May). Automatic behavior sensing for a bomb-detecting dog. In Unmanned Systems Technology XVII (Vol. 9468, p. 94680K). International Society for Optics and Photonics.

Ochoa, M., Bohórquez, G \& Rojas-Guevara, J. (2020). II Congreso latinoamericano de comportamiento y bienestar animal (ISAE). Ponencia: vínculo humano-animal: morfología, fin zootécnico-comportamental y salud del perro de pastoreo del desierto colombiano. Relaciones actuales entre humanos y animales. Medellín, Colombia.

Pavlov, I. P. (1926). Edited and translated by GV Anrep Conditioned reflexes: An Investigation of the Physiological Activity of the Cerebral Cortex.

Pearce, J. M. (20I3). Animal learning and cognition: an introduction. Psychology Press.

Policía Nacional de Colombia (PNC) (20I5). Tras las huellas de los guías caninos de la Policía Nacional. Escuela de Guías y Adiestramiento Canino de la Policía Nacional de Colombia https://www.policia.gov.co/file//23753/ download?token=d7IIU_CX.

Polsky, R. H. (1994). Electronic shock collars: are they worth the risks? Journal of the American Animal Hospital Association, 30(5), 463-468.

Polsky, R. H. (1994). Electronic shock collars: are they worth the risks? Journal of the American Animal Hospital Association, 30(5), 463-468.

Prada-Tiedemann, P., Ochoa-Torres, M., RojasGuevara, J., \& Bohórquez, G. (2019a). Incidencia de la discriminación de olor en el entrenamiento de los equipos caninos detectores de sustancias: impacto de su evaluación para la certificación final. Revista Logos Ciencia \& Tecnología, I2(I). https://doi. org/l 0.22335/rlct.978-958-52080-7-0.

Prada-Tiedemann, P., Rojas-Guevara, J., Bohórquez, G., Ochoa-Torres, M., \& Córdoba-Parra, J. (20/9b). Los equipos caninos policiales: importancia de los criterios de selección, entrenamiento, certificación y rendimiento. Policía Nacional de Colombia. http://dx.doi. org/l 0.22335/rlct.978-958-52080-7-0.

Raymer, J., Rojas-Guevara, J., \& Prada-Tiedemann, P. (2020). Decomposition residual odor volatiles in soil from a west texas environment. Revista Criminalidad, 62(3): 79-I0I. http:// www.scielo.org.co/scielo.php?pid=SI 7943 I 082020000300079\&script=sci_abstract\& tlng=es.

Riepl, M. (20I3). Characteristics of electronic training collars for dogs. Veterinary Record, 172(9), 242-243.
Rodríguez, M. B., Zafra, S. L., \& Ortega, S. P. Q. (20I5). La revisión sistemática de la literatura científica y la necesidad de visualizar los resultados de las investigaciones. Revista Logos, Ciencia \& Tecnología, 7(I), I01-103.

Rojas-Guevara, J.U., Prada-Tiedemann, P.A., Titus, K., Córdoba-Parra, J.D., \& Bohórquez, G.A (2020). Forensic studies with human analogues: evaluation of decomposition and the use of residual odor detection dogs. National Police of Colombia. https://doi.org//0.22335/ EDNE.I0.

Rojas-Guevara, J. U., Córdoba-Parra, J. D., Bohórquez, G. A., Vega-Contreras, R. A., \& PradaTiedemann, P. (202 la). El papel de los equipos caninos detectores de restos humanos en la investigación criminal. Revista Científica General José María Córdova, 19(33), 91-III. https://doi.org/10.21830/19006586.638.

Rojas-Guevara, J., Bohórquez, G., Carrascal, J., \& Tiedemann, P. (202lb). II Congreso latinoamericano de comportamiento y bienestar animal (ISAE). Ponencia: Especificidad de la prueba doble ciego para la certificación de los perros detectores de fauna silvestre. Relaciones actuales entre humanos y animales. Medellín, Colombia. Rev. Fac. Nac. Agron. Medellín (202I), 74(Suplemento), S169-185.

Rojas-Guevara, J Vega-Contreras, R, Bohórquez, G., \& Carrascal, J. (202/c). II Congreso latinoamericano de comportamiento y bienestar animal (ISAE). Ponencia: Evaluación del primer perro detector de oro: una de las estrategias para impactar el comercio ilegal. Estrategias en educación en comportamiento y bienestar. Medellín, Colombia. Rev. Fac. Nac. Agron. Medellín (202I), 74 (Suplemento), SI69-185.

Rooney, N. J., \& Cowan, S. (20I I). Training methods and owner-dog interactions: Links with dog behaviour and learning ability. Applied Animal Behaviour Science, 132(3-4), I69-I77.

Sánchez, W., Sandoval, M., Bohórquez, G., \& Zapata, C. (20|4). Entrenamiento y uso de caninos detectores de fauna silvestre en la Policía Nacional. Editorial: Escuela de Guías y Adiestramiento Canino de la Policía Nacional de Colombia. ISBN. 978-958-99407-2-3. https://www. policia.gov.co/file/ I 2377 I/download?token $=X 2$ amWDQf.

Salgirli, Y., Schalke, E., Boehm, I., \& Hackbarth, H. (20I2). Comparison of learning effects and stress between 3 different training methods (electronic training collar, pinch collar and quitting signal) in Belgian Malinois Police Dogs. Revue De Medecine Veterinaire, I63, 530-535. 
Sarmiento-Hernández, E. A., Rojas-Guevara, J. U., \& Rojas Guevara, P. J. (2020). Incidencia del proceso de paz con las FARC en la política antidrogas de Colombia. Revista Científica General José María Córdova, I8(32), 817-837. https://doi.org/ 0.2 I830//9006586.632

Schalke, E., Stichnoth, J., Ott, S., \& Jones-Baade, R. (2007). Clinical signs caused by the use of electric training collars on dogs in everyday life situations. Applied Animal Behaviour Science, 105(4), 369-380.

Schalke, E., Ott, S., \& Hackbarth, H. (2008). Training methods in dogs with and without electric training collars. DTW. Deutsche tierarztliche Wochenschrift, I I5(4), I40-I42.

Schilder, M. B., \& van der Borg, J. A. (2004). Training dogs with help of the shock collar: short and long term behavioural effects. Applied Animal Behaviour Science, 85(3-4), 319-334.

Secretaría Distrital de Seguridad, Convivencia y Justicia (SDSCJ). (2020). Delitos de alto impacto. Recuperado de: http://analitica.scj. gov.co/analytics/saw.dll?Portal.

Silver, Z. A., Furlong, E. E., Johnston, A. M., \& Santos, L. R. (202I). Training differences predict dogs' (Canis lupus familiaris) preferences for prosocial others. Animal cognition, 24(I), 75-83. https://doi.org/10.1007/s 1007/-020$014 \mid 7-9$.

Skinner, B. F., (1938). The Behavior of Organisms: An Experimental Analysis. Appleton-Century, Oxford, England.
Skinner, B. F. (197I). Operant conditioning. The encyclopedia of education, 7, 29-33.

Smith, S. M., \& Davis, E. S. (2008). Clicker increases resistance to extinction but does not decrease training time of a simple operant task in domestic dogs (Canis familiaris). Applied Animal Behaviour Science, I I0(3-4), 318-329.

Snyder, H. (2019). Literature review as a research methodology: An overview and guidelines. Journal of Business Research, 104, 333-339.

Strychalski, J., Gugołek, A., \& Konstantynowicz, M. (2015). Clicker training efficiency in shaping the desired behaviour in the following dog breeds: boxer, chow chow and yorkshire terrier. Polish Journal of Natural Sciences, 30(3), 235-243.

Tolman, E. C. (1925). Purpose and cognition: the determiners of animal learning. Psychological Review, 32(4), 285.

Villarreal, C., Rojas, J., \& Ochoa, M. (2018). Impacto operacional de los equipos caninos en la lucha contra el narcotráfico en Colombia: una revisión de la contribución, estrategias y tendencias recientes. Revue Scientifique et Technique, 37(I), 189-200. https://doi. org/I0.20506/rst.37.I.275I. 